\title{
Optimization of Joint Power and Bandwidth Allocation in Multi-Spot-Beam Satellite Communication Systems
}

\author{
Heng Wang, Aijun Liu, and Xiaofei Pan \\ College of Communications Engineering, PLA University of Science \& Technology, Nanjing, Jiangsu 210007, China \\ Correspondence should be addressed to Heng Wang; wangheng0987654321@126.com
}

Received 30 August 2013; Accepted 5 December 2013; Published 12 January 2014

Academic Editor: Jun Jiang

Copyright ( 2014 Heng Wang et al. This is an open access article distributed under the Creative Commons Attribution License, which permits unrestricted use, distribution, and reproduction in any medium, provided the original work is properly cited.

\begin{abstract}
Multi-spot-beam technique has been widely applied in modern satellite communication systems. However, the satellite power and bandwidth resources in a multi-spot-beam satellite communication system are scarce and expensive; it is urgent to utilize the resources efficiently. To this end, dynamically allocating the power and bandwidth is an available way. This paper initially formulates the problem of resource joint allocation as a convex optimization problem, taking into account a compromise between the maximum total system capacity and the fairness among the spot beams. A joint bandwidth and power allocation iterative algorithm based on duality theory is then proposed to obtain the optimal solution of this optimization problem. Compared with the existing separate bandwidth or power optimal allocation algorithms, it is shown that the joint allocation algorithm improves both the total system capacity and the fairness among spot beams. Moreover, it is easy to be implemented in practice, as the computational complexity of the proposed algorithm is linear with the number of spot beams.
\end{abstract}

\section{Introduction}

In recent years, the multi-spot-beam technique has played an important role in the satellite communication systems, as it can not only supply higher power density to a particular spot beam but also construct flexible service networks. However, the satellite power and bandwidth resources are scarce and expensive in multi-spot-beam satellite communication systems. As a result, it is crucial to make effort to enhance the utilization efficiency of power and bandwidth resource.

Because the coverage of each spot beam is different, the real traffic demand of each spot beam is different and time varying. In addition, the channel condition of each spot beam is also affected by the weather condition. Therefore, it is important to dynamically allocate the bandwidth and power resource to each spot beam to meet its traffic demand.

In the previous work, separate optimal power or bandwidth allocation algorithms were proposed in [1-4] and [5], respectively. The work in [1] emphasized the mathematical formulation and analytic solutions of the optimum power resource allocation problem and explained the tradeoff between the total system capacity and fairness among all spot beams with different traffic demands and delay constraints.
The optimization problem is solved based on the KarushKuhn-Tucker (KKT) condition. However, the works in [1] did not provide the way to find the optimal Lagrangian multipliers. Therefore, the methods of bisection and subgradient were applied to search for the optimal Lagrangian multipliers in [2, 3]. To improve the total system capacity, a method of selecting small number of active spot beams was proposed in [4], while keeping the fairness among spot beams. The optimal power allocation algorithms failed to consider one problem that the optimal power allocation algorithm required the satellite power amplifier to operate with high back-off, degrading the system performance. To solve the problem, in [5] a dynamic bandwidth allocation algorithm was proposed. The above works proved that it was needed to allocate more resources to the spot beam with higher traffic demand to get fairness among spot beams; thus the total system capacity decreased, due to the concavity of the capacity function with a fixed power or bandwidth allocation. To overcome this drawback, in this paper we propose a joint bandwidth and power allocation algorithm.

For completeness, it is noted that the joint bandwidth and power allocation problem has been investigated for terrestrial wireless communication systems [6-8]. In [6], 
the joint bandwidth and power allocation problem in downlink transmission was investigated. In [7], an optimal joint bandwidth and power allocation algorithm in wireless multiuser networks with and without relaying was proposed. In [8], a joint power and bandwidth allocation algorithm with QoS support in heterogeneous wireless networks was proposed, using convex optimization methodology. However, the conclusions obtained in the above works cannot be applied to the multi-spot-beam satellite communication system, due to the fact that the constraints in the system we investigate are different from those in the systems mentioned above.

In this paper, we propose solving the problem of joint bandwidth and power allocation for the multi-spot-beam satellite communication system. We initially formulate the problem of joint bandwidth and power allocation as a nonlinear optimization problem and prove the optimization problem is convex. The object of optimization problem is to match the capacity allocated to each spot beam to the traffic demand as closely as possible, taking into account a compromise between the maximum total system capacity and the proportional fairness among spot beams. A joint bandwidth and power allocation iterative algorithm based on duality theory is then proposed to obtain the optimal solution of this optimization problem. Compared with the separate power or bandwidth optimal allocation algorithm, the proposed joint bandwidth and power allocation algorithm improves the total system capacity and the fairness among spot beams. In addition, we discuss the impact of traffic demand, channel condition, and the delay constraint of each spot beam on the allocation results.

The remainder of this paper is organized as follows. In Section 2, we formulate the optimization problem of joint bandwidth and power allocation and prove the optimization problem is convex. Section 3 proposes the optimal joint bandwidth and power allocation algorithm based on duality theory, and Section 4 presents the simulation results and analyzes the impact of traffic demand, channel conditions, and delay constraints on the allocation result. Section 5 concludes the paper.

\section{Mathematical Formulation of Joint Bandwidth and Power Allocation}

2.1. Modeling of Downlink Multi-Spot-Beam Capacity. Figure 1 shows the system configuration of a multi-spotbeam satellite communication system, where the traffic demand of the $i$ th spot beam is $T_{i}$, the power allocated to the $i$ th spot beam is $P_{i}$, the bandwidth allocated to the $i$ th spot beam is $W_{i}$, and the signal attenuation factor of the $i$ th spot beam is $\alpha_{i}^{2}$. It is noted that $\alpha_{i}^{2}$ mainly consists of the effects of weather conditions, free space loss, and antenna gain.

Using time sharing for Gaussian broadcast channels [9], the Shannon bounded capacity $C_{i}$ for the $i$ th spot beam is given as

$$
C_{i}=W_{i} \log _{2}\left(1+\frac{\alpha_{i}^{2} P_{i}}{W_{i} N_{0}}\right)
$$

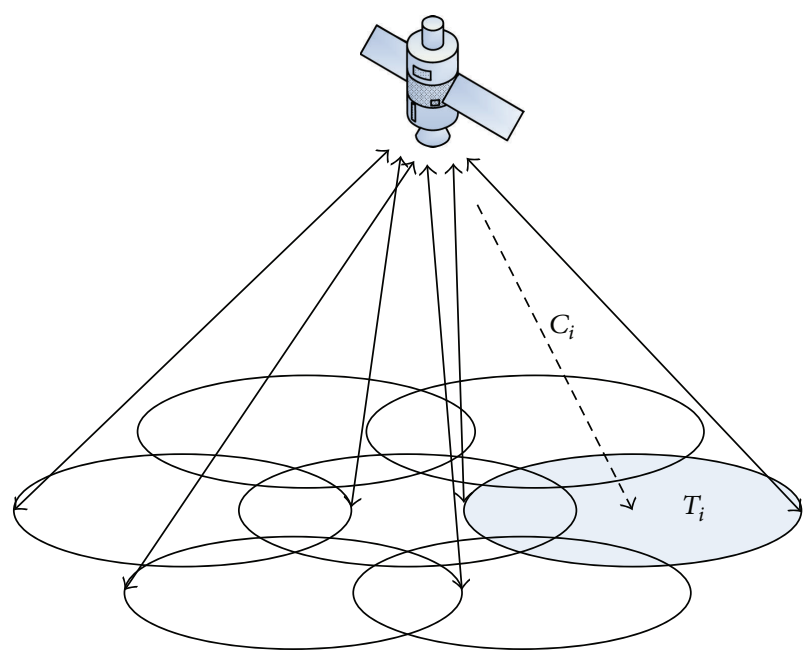

FIGURE 1: System configuration of multi-spot-beam satellite system.

where $N_{0}$ is the noise power density of each spot beam. It is noted that interbeam interference from the sidelobes of adjacent spot beams degenerates the Shannon capacity. However, in this paper, we ignore interbeam interference, because we consider very narrow spot beams over a large number of spot beams [1]. It is observed from (1) that the spot beam capacity $C_{i}$ is increased as the bandwidth or power allocated to the spot beam increases. However, the total bandwidth and power of the satellite are fixed, so the capacity of the system is limited.

If the total system power and bandwidth resources are sufficient to support the total traffic demand generated by all the spot beams, it seems to be meaningless for us to make efforts to improve the resource utilization efficiency. Therefore we only focus on the scenario where the total traffic demand exceeds the total available system capacity [1].

2.2. Delay Constraint. In this section, we describe the impact of the delay constraint. In the practical multi-spot-beam satellite system, there is much real-time traffic, such as video and audio flows, for which the delay performance is an important evaluation criterion. According to the analysis in [1], the delay constraint of each spot beam can be transformed into the traffic demand of each spot beam, which is given as follows:

$$
C_{i} \geq \frac{T_{i}}{\left(1-e_{i}\right) D_{i}},
$$

where $e_{i}$ is the packet error rate over the link of the $i$ th spot beam, $D_{i}$ is the average delay deadline of the $i$ th spot beam, and $\left(1-e_{i}\right) D_{i}>1$. The constraint (2) implies that each spot beam has a minimal traffic demand, which is determined by $e_{i}$ and $D_{i}$. It is seen that both the higher priority traffic with a smaller $D_{i}$ and the worse channel condition with a larger $e_{i}$ will lead to a larger fraction of the capacity.

2.3. Optimization Problem Formulation. There are many metrics to evaluate the system performance, and different 
metrics may lead to different allocation results. Therefore, it is very important for us to choose an appropriate metric. In this paper, the metric is to minimize the deficit between the traffic demand and the capacity allocated, taking into account a compromise between the maximum total system capacity and the proportional fairness among spot beams. Motivated by Choi and Chan [1], the problem can be formulated by using the $n$th order objective function as follows:

$$
\begin{array}{ll}
\min _{\left\{P_{i}\right\},\left\{W_{i}\right\}} & \sum_{i=1}^{N}\left(T_{i}-C_{i}\right)^{n} \\
\text { s.t. } \quad & C_{i}=W_{i} \log _{2}\left(1+\frac{\alpha_{i}^{2} P_{i}}{W_{i} N_{0}}\right) \leq T_{i} \quad \forall i \\
& \sum_{i=1}^{N} P_{i} \leq P_{\text {total }} \\
& \sum_{i=1}^{N} W_{i} \leq W_{\text {total }}, \\
& C_{i}=W_{i} \log _{2}\left(1+\frac{\alpha_{i}^{2} P_{i}}{W_{i} N_{0}}\right) \geq \frac{T_{i}}{\left(1-e_{i}\right) D_{i}} \quad \forall i
\end{array}
$$

where $n$ is integer and $n \geq 2$. When $n=1$, the problem is equivalent to maximizing the total system capacity and the fairness among spot beams is ignored. So we ignore the case with $n=1$. The constraint (4) indicates that the allocated resource should not exceed the traffic demand of each spot beam. Conditions (5), (6), and (7) imply the constraint for the total power, the total bandwidth, and the delay, respectively.

The above optimization is a nonlinear programming problem. It is found that the optimal variables $W_{i}$ and $P_{i}$ are coupled in the problem above, which makes the global optimal solution difficult to be obtained. To make the above problem tractable, in the following section, we propose a joint power and bandwidth allocation algorithm based on duality optimization theory [10-13]. It is known that if the optimization problem is convex, the duality gap is zero. As a result, the optimal solution obtained by the duality problem is the global optimal solution of the primary problem [14]. Fortunately, the optimization problem mentioned above is convex, the proof of which is shown in the appendix.

\section{Proposed Optimal Joint Bandwidth and Power Allocation Algorithm}

As mentioned above, the joint bandwidth and power allocation algorithm is based on the duality theory. By introducing nonnegative dual variables $\mu, \lambda$, and $\boldsymbol{\rho}=\left[\rho_{1}, \rho_{2}, \ldots, \rho_{n}\right]$, the Lagrange function is yielded, given as

$$
\begin{aligned}
L(\mathbf{P}, \mathbf{W}, \boldsymbol{\rho}, \lambda, \mu)= & \sum_{i=1}^{N}\left[T_{i}-W_{i} \log _{2}\left(1+\frac{\alpha_{i}^{2} P_{i}}{W_{i} N_{0}}\right)\right]^{n} \\
& -\mu\left(W_{\text {total }}-\sum_{i=1}^{N} W_{i}\right)
\end{aligned}
$$

$$
\begin{aligned}
& -\sum_{i=1}^{N} \rho_{i}\left[C_{i}-\frac{T_{i}}{\left(1-e_{i}\right) D_{i}}\right] \\
& -\lambda\left(P_{\text {total }}-\sum_{i=1}^{N} P_{i}\right)
\end{aligned}
$$

where $\mathbf{P}=\left[P_{1}, P_{2}, \ldots, P_{n}\right]$ and $\mathbf{W}=\left[W_{1}, W_{2}, \ldots, W_{n}\right]$.

From (8), Lagrange dual function can be obtained by

$$
D(\boldsymbol{\rho}, \lambda, \mu)=\min _{\mathbf{P}, \mathbf{W}} L(\mathbf{P}, \mathbf{W}, \boldsymbol{\rho}, \lambda, \mu)
$$

and the dual problem can be written as

$$
d^{*}=\max _{\lambda \geq 0, \mu \geq 0, \rho \geq 0} D(\rho, \lambda, \mu) .
$$

According to [10], the dual problem in (10) can be further decomposed into the following three sequentially iterative subproblems.

Subproblem 1. Power Allocation. Given the dual variables $\mu, \lambda$, and $\rho$, for any $i=[1, \ldots, N]$, maximizing (8) with respect to $P_{i}$ brings the equation

$$
\begin{aligned}
& \frac{n \alpha_{i}^{2} W_{i}}{\ln 2\left(W_{i} N_{0}+\alpha_{i}^{2} P_{i}^{*}\right)} \\
& \quad \times\left\{\left[T_{i}-W_{i} \log _{2}\left(1+\frac{\alpha_{i}^{2} P_{i}^{*}}{W_{i} N_{0}}\right)\right]^{n-1}+\frac{\rho_{i}}{n}\right\}=\lambda .
\end{aligned}
$$

The $P_{i}^{*}$ can be obtained from (11) by numerical calculation methods, for example, the golden section method. The optimized power allocation of the $i$ th spot beam $P_{i}^{\text {opt }}=$ $\max \left\{0, P_{i}^{*}\right\}$.

Subproblem 2. Bandwidth Allocation. Substituting the $P_{i}^{\text {opt }}$ into (8), maximizing (8) with respect to $W_{i}$ brings the following equation:

$$
\begin{aligned}
& {\left[\left(T_{i}-C_{i}^{*}\right)^{n-1}+\frac{\rho_{i}}{n}\right]} \\
& \quad \times\left\{\frac{C_{i}^{*}}{W_{i}^{*}}-\frac{1}{\ln 2\left[N_{0} W_{i}^{*} /\left(\alpha_{i}^{2} P_{i}^{\mathrm{opt}}\right)+1\right]}\right\}=\frac{\mu}{n},
\end{aligned}
$$

where $C_{i}^{*}=W_{i}^{*} \log _{2}\left(1+\left(\alpha_{i}^{2} P_{i}^{\mathrm{opt}} / W_{i}^{*} N_{0}\right)\right)$.

From (12), we can obtain the $W_{i}^{*}$ by using golden section method. The optimized bandwidth allocated to the $i$ th spot beam $W_{i}^{\text {opt }}=\max \left\{0, W_{i}^{*}\right\}$.

Subproblem 3. Dual Variables Update. The optimal dual variables can be obtained by solving its dual problem

$$
\left(\boldsymbol{\rho}^{\mathrm{opt}}, \lambda^{\mathrm{opt}}, \mu^{\mathrm{opt}}\right)=\arg \min _{\lambda, \mu, \boldsymbol{\rho}} \max \left\{L\left(\mathbf{P}^{\mathrm{opt}}, \mathbf{W}^{\mathrm{opt}}, \boldsymbol{\rho}, \lambda, \mu\right)\right\} .
$$


Because the dual function is always convex, here we can use a subgradient (a generalization of gradient) update method as follows [11]:

$$
\begin{aligned}
& \lambda^{n+1}=\left[\lambda^{n}-\Delta_{\lambda}^{n}\left(P_{\text {total }}-\sum_{i=1}^{N} P_{i}^{\mathrm{opt}}\right)\right]^{+}, \\
& \mu^{n+1}=\left[\mu^{n}-\Delta_{\mu}^{n}\left(W_{\text {total }}-\sum_{i=1}^{N} W_{i}^{\mathrm{opt}}\right)\right]^{+}, \\
& \rho_{i}^{n+1}=\left[\rho_{i}^{n}-\Delta_{\rho}^{n}\left(C_{i}^{\mathrm{opt}}-\frac{T_{i}}{\left(1-e_{i}\right) D_{i}}\right)\right]^{+},
\end{aligned}
$$

where $[x]^{+}=\max \{0, x\}, n$ is the iteration number, and $\Delta$ is the iteration step size.

The above dual variable updating algorithm is guaranteed to converge to the optimal solution, as long as the iteration step chosen is sufficiently small [11].

The whole process of the proposed joint bandwidth and power allocation algorithm can be summarized as follows.

The Proposed Joint Bandwidth and Power Allocation Algorithm

Step 1. Set appropriate initial values for the dual variables and the bandwidth of each spot beam.

Step 2. Substitute the values of the bandwidth of each spot beam and the dual variables into (11) and then calculate the optimized power allocated to each spot beam.

Step 3. Substitute into (12) both the power values for each spot beam obtained from Step 2 and the Lagrangian multipliers, calculate the optimized bandwidth allocated to each user, and then calculate the optimized bandwidth allocated to each spot beam.

Step 4. Substitute the values of the power and bandwidth of each spot beam which are separately obtained from Steps 2 and 3 into (14) and then update the dual variables.

Step 5. If the conditions of $\left|\lambda^{n+1}\left(P_{\text {total }}-\sum_{i} P_{i}\right)\right|<\varepsilon$, $\left|\mu^{n+1}\left(W_{\text {total }}-\sum_{i} W_{i}^{\text {opt }}\right)\right|<\varepsilon$, and $\mid \rho_{i}^{n+1}\left(C_{i}^{\text {opt }}-T_{i} /[(1-\right.$ $\left.\left.\left.e_{i}\right) D_{i}\right)\right] \mid<\varepsilon, \forall i$, are satisfied simultaneously, then terminate the algorithm. Otherwise, jump to Step 2.

According to the above process, it is seen that the computational complexities of Steps 2, 3, and 4 are $O(S N)$, $O(S N)$, and $O(2+N)$, where $N$ is the number of the spot beams and $S$ is the computational complexity of the golden section method. Therefore, the total computational complexity of the proposed algorithm is $\mathrm{O}(2 \mathrm{KSN}+2 \mathrm{~K}+$ $K N$ ), where $K$ is the number of the iterations. It is noted that both $K$ and $S$ are independent of $N$. As a result, the computational complexity of the proposed algorithm is linear with the number of the spot beams, which indicates that the algorithm can be implemented in practice.

\section{Simulation Results and Analysis}

For the simulation, a Ka band multi-spot-beam satellite communication system model is set up. The system has 10 spot beams, the total power of the satellite is $200 \mathrm{~W}$, and the total bandwidth of the satellite is $500 \mathrm{MHz}$. The traffic demand of each spot beam increases from $80 \mathrm{Mbps}$ to $260 \mathrm{Mbps}$ by steps of $20 \mathrm{Mbps}$. The minimal traffic demand of each spot beam caused by the delay constraint is $20 \%$ of traffic demand.

4.1. Convergence Behavior of Proposed Joint Allocation Algorithm. To show the convergence behavior of the proposed joint allocation algorithm, we first assume that the channel condition of each spot beam is the same and the normalized noise power spectral density parameter $N_{0} / \alpha_{i}^{2}$ is $0.2 e^{-6}$. Moreover, the order of the objective function is 2 .

Figures 2 and 3 show the convergence of the dual variables and optimal variables. As seen in Figure 2, each dual variable has its own update step size, and the dual variables converge to their optimal value after finite iterations, which depend on the choice of the step sizes. When the dual variables converge, it is noted that from Figure 3 the optimal variables also converge.

4.2. Efficiency of the Proposed Joint Allocation Algorithm. To verify the resource efficiency of the proposed optimal joint allocation algorithm (OBOP), we compare it with the following three algorithms.

(a) Uniform bandwidth allocation and uniform power allocation (UBUP): UBUP algorithm divides the bandwidth and power resource equally between each spot beam.

(b) Uniform bandwidth allocation and optimal power allocation (UBOP) [1]: UBOP algorithm divides the bandwidth resource equally between each spot beam and optimizes the power allocation for each spot beam.

(c) Uniform power allocation and optimal bandwidth allocation (OBUP) [5]: OBUP algorithm divides the power resource equally between each spot beam and optimizes the bandwidth allocation for each spot beam.

When the channel conditions are the same, Figure 4 shows the capacity distributions of spot beams which are allocated by the four algorithms. Table 1 shows the total system capacity of the four algorithms. As shown in Figure 4, to get the fairness among spot beams, the separate optimal allocation algorithms (UBOP and OBUP) will provide more capacity to higher demand spot beams. It is known that the capacity does not linearly increase with the power or bandwidth increasing, due to the concavity of the capacity function with a fixed bandwidth or power allocation. This results in a lower total system capacity. However, in the proposed joint allocation algorithm (OBOP), the capacity function is almost linear, since the bandwidth and power are jointly dynamically allocated. As a result, the total system capacity is much improved compared to the separate optimal 


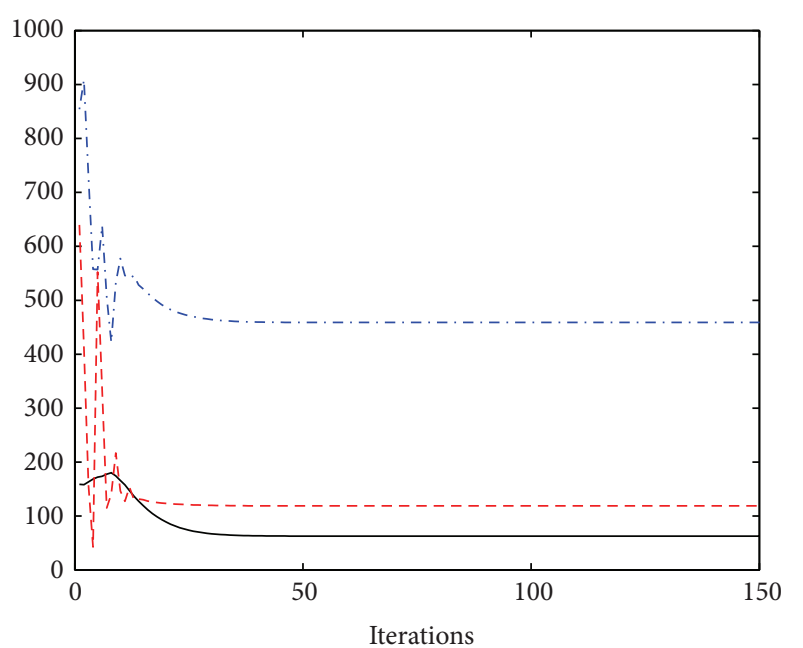

$\rho(1)$ (step size is 0.4 )

-. - $\lambda$ (step size is 1.5 )

$---\mu$ (step size is 0.7$)$

Figure 2: The convergence behavior of the dual variables.

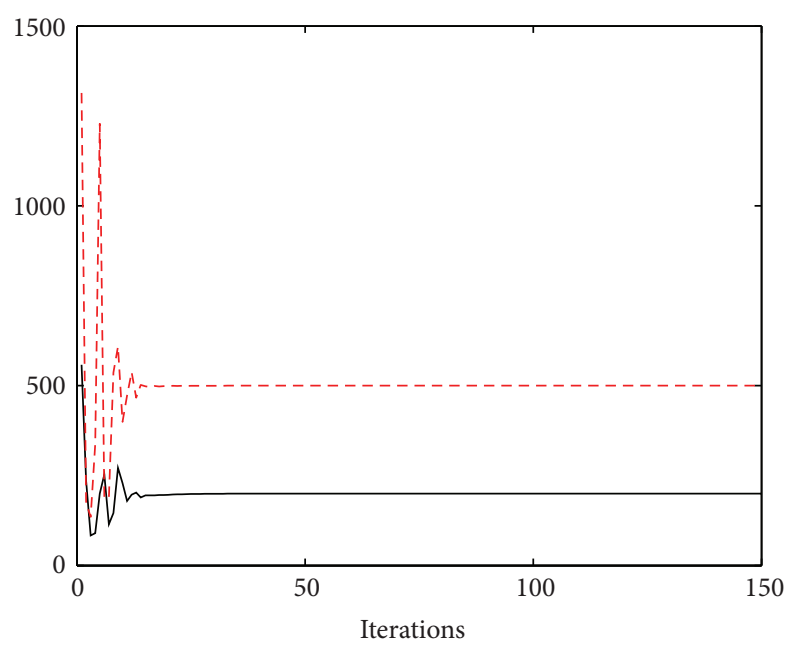

- The sum of the power allocated to each beam

- - - The sum of the bandwidth allocated to each beam

FIGURE 3: The convergence behavior of the optimal variables.

TABLE 1: The total system capacity of the four algorithms when the channel conditions are the same.

\begin{tabular}{lc}
\hline Algorithms & $\sum C_{i}$ \\
\hline UBUP & $792.48125 \mathrm{Mbps}$ \\
UBOP [1] & $748.83866 \mathrm{Mbps}$ \\
OBUP [5] & $756.15281 \mathrm{Mbps}$ \\
The proposed OBOP & $792.48124 \mathrm{Mbps}$ \\
\hline
\end{tabular}

allocation algorithm. This conclusion can be also concluded from Table 1 .

When the channel conditions are the same, Figure 5 shows the deficit between the traffic demand and the capacity

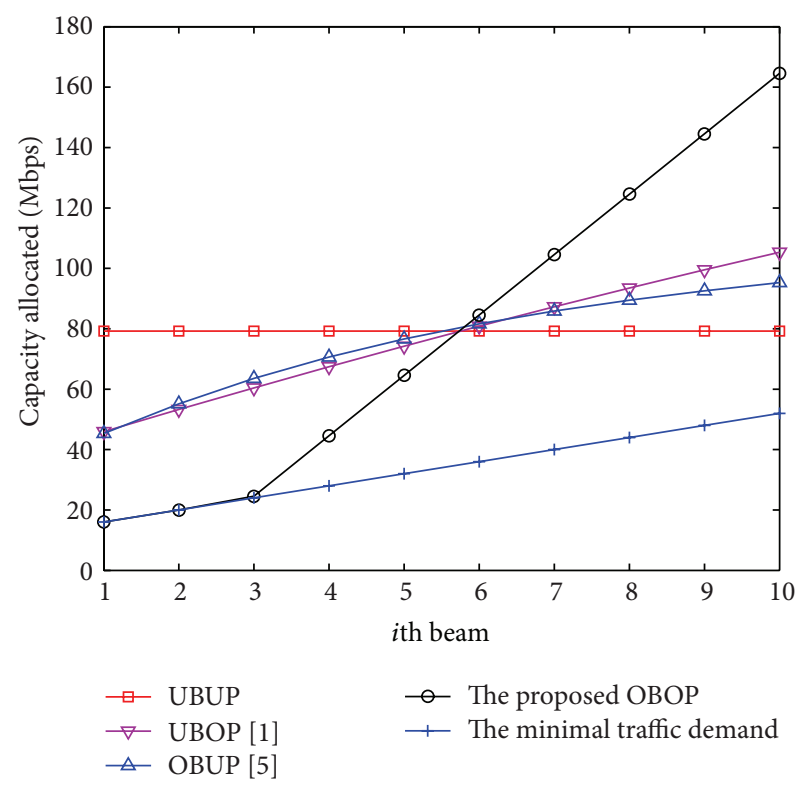

Figure 4: Comparison of the four algorithms in terms of the capacity allocated to each spot beam when channel conditions are the same.

TABLE 2: The total sum of $\left(T_{i}-C_{i}\right)^{2}$ of the four algorithms when the channel conditions are the same.

\begin{tabular}{lc}
\hline Algorithms & $\sum\left(T_{i}-C_{i}\right)^{2}$ \\
\hline UBUP & $115.36 E 15$ \\
UBOP [1] & $101.70 E 15$ \\
OBUP [5] & $106.76 E 15$ \\
The proposed OBOP & $83.366 E 15$ \\
\hline
\end{tabular}

allocated to each spot beam. Table 2 presents the sum of the deficit between the traffic demand and the capacity allocated to each spot beam. From Figure 5, it is shown that in the joint allocation algorithm (OBOP), from the spot beam 3 to spot beam 10, the deficit between traffic demand and capacity allocated is almost the same, so the fairness among spot beams is better than the separate optimal allocations (UBOP and OBUP). This conclusion can be also observed from Table 2, since the deficit between the traffic demand and the capacity allocated is smaller than the separate optimal allocations. Together with the conclusion above regarding total system capacity, we can conclude that the performance of the optimal joint allocation algorithm (OBOP) is much improved compared with the separate optimal algorithms (UBOP and OBUP).

4.3. Impact of the Channel Condition and Delay Constraints. We compare the allocation result of the OBOP allocation with delay constraint with that of the OBOP allocation without the delay constraint (OBOPND) to analyze the impact of the delay constraints. When there is no delay constraint on each spot beam, the allocation result can be obtained by the same algorithm, where we just need to remove the dual variable $\rho$ in (8). 


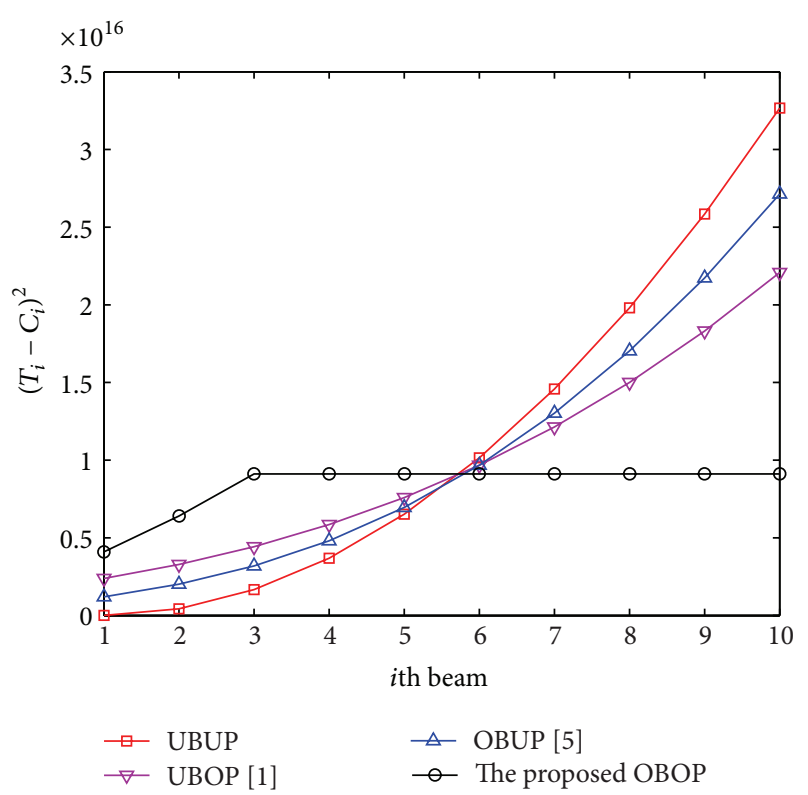

FIGURE 5: Comparison of the four algorithms in terms of the deficit between the traffic demand and the capacity allocated to each spot beam when channel conditions are the same.

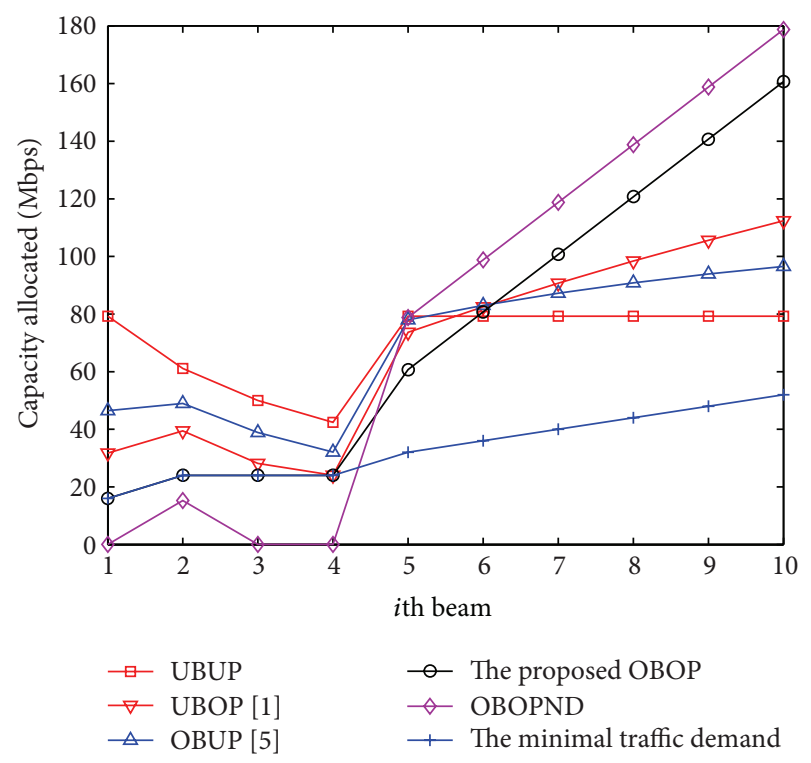

FIgURE 6: The capacity allocated by five algorithms to each spot beam when channel conditions are not the same.

From spot beam 2 to spot beam 4, we set the normalized noise power spectral density parameters $N_{0} / \alpha_{i}^{2}$ to $0.3 e^{-6}$, $0.4 e^{-6}$, and $0.5 e^{-6}$, respectively, to analyze the impact of channel condition on the allocation results. Each traffic demand of the three spot beams is $120 \mathrm{Mbsp}$, so the total traffic demand is the same as the above simulation scenario. The order of the objective function also is 2 .

When the channel conditions are not the same, Figure 6 shows the capacity distributions of spot beams which are allocated by the 5 algorithms. Table 3 shows the total system
TABLE 3: The total system capacity of the five algorithms when the channel conditions are not the same.

\begin{tabular}{lc}
\hline Algorithms & $\sum C_{i}$ \\
\hline UBUP & $708.26 \mathrm{Mbps}$ \\
UBOP [1] & $698.05 \mathrm{Mbps}$ \\
OBUP [5] & $695.87 \mathrm{Mbps}$ \\
The proposed OBOP & $752.16 \mathrm{Mbps}$ \\
OBOPND & $788.06 \mathrm{Mbps}$ \\
\hline
\end{tabular}

TABLE 4: The total sum of $\left(T_{i}-C_{i}\right)^{2}$ of the 5 algorithms when the channel conditions are not the same.

\begin{tabular}{lr}
\hline Algorithms & $\sum\left(T_{i}-C_{i}\right)^{2}$ \\
\hline UBUP & $123.97 E 15$ \\
UBOP [1] & $112.25 E 15$ \\
OBUP [5] & $114.08 E 15$ \\
The proposed OBOP & $90.919 E 15$ \\
OBOPND & $85.730 E 15$ \\
\hline
\end{tabular}

capacity of the 5 algorithms. From Figure 6, it is shown that both the separate optimal allocation algorithm and the proposed joint allocation algorithm allocate more capacity to the spot beam with better channel condition than that with worse channel condition; especially for the OBOPND algorithm, no capacity is allocated to spot beam 3 and spot beam 4 . Therefore, the metric we choose in this paper not only considers the fairness among spot beams, but also tries to maximize the throughput of the system, which achieves a good system performance as we expected. Due to the delay constraint, we have to allocate a minimal traffic to spot beams with worse channel condition; thus, the total system capacity decreases. This conclusion is also shown in Table 3. So we conclude that when the channel condition of each spot beam is not the same, the delay constraint of each spot beam decreases the total system capacity.

When the channel conditions are not the same, Figure 7 shows the deficit between the traffic demand and the capacity allocated to each spot beam. Table 4 presents the sum of the deficits between the traffic demand and the capacity allocated to each spot beam. It is shown that the worse channel conditions and the delay constraint decrease the fairness among spot beams.

4.4. Impact of the Order of the Objective Function and Delay Constraints. As mentioned in Section 2.3, different metrics may lead to different allocation results. Here we analyze the impact of the order of the objective function. The channel condition of each spot beam is set to be the same.

Figure 8 shows the allocated capacity of each spot beam and Table 5 shows the total system capacity when the order of the objective function is different.

When there is no delay constraint in each spot beam, as we use a higher-order deviation objective function, more resources are provided for higher traffic demand spot beams, while a lower-order cost function gives relatively more resource to lower traffic demand spot beams. For example, 


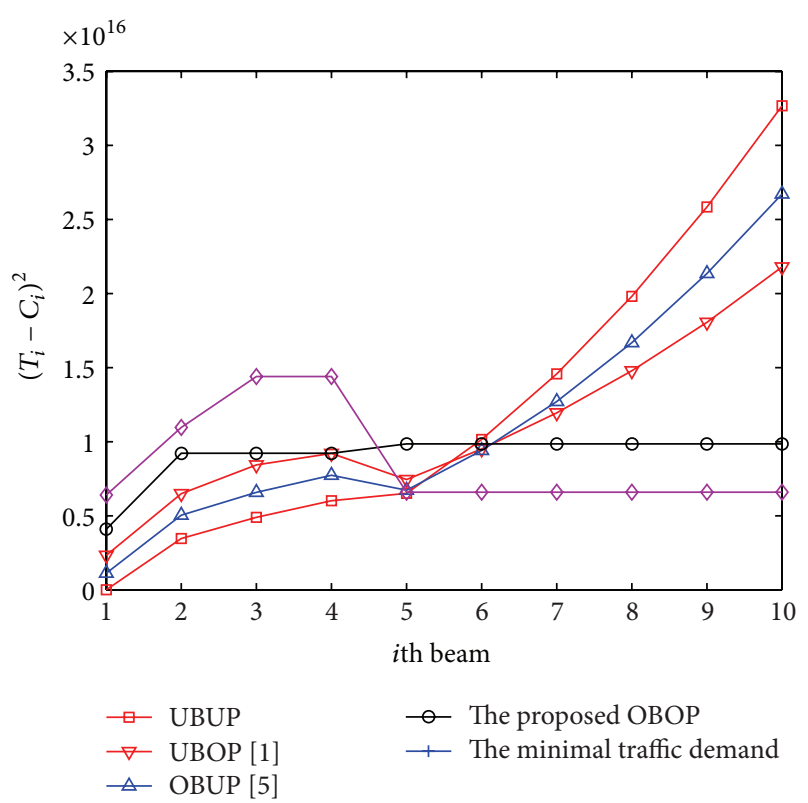

FIGURE 7: The deficit between the traffic demand and the capacity allocated by 5 algorithms to each spot beam when channel conditions are not the same.

the cubic objective function provides no capacity for the three lowest traffic demand spot beams. Although the capacity allocated to each spot beam is different, the total system capacities of different objective function are almost the same.

When there is delay constraint in each spot beam, a minimal traffic demand must be allocated to them. As a result, for the same cubic objective function, the three lowest traffic demand spot beams are allocated to the minimal traffic demand. It is also seen that the capacity allocated to each spot beam is the same by using second- and third-order of the objective function.

\section{Conclusion}

In the multi-spot-beam satellite system, due to the scarceness of the satellite resource, it is crucial for us to improve the resource utilization efficiency. To this end, in this paper we first formulated the joint bandwidth and power allocation problem as a convex optimization problem. Then we proposed a joint bandwidth and power allocation algorithm based on duality theory to get the optimal solution. Compared with the individual optimal power or bandwidth allocation, the proposed joint optimal bandwidth and power allocation algorithm improved the total system capacity and the fairness among spot beams, while the computational complexity of the algorithm was linear with the number of spot beams. Therefore, the proposed algorithm can be implemented in practice.

It was shown from the simulation results that the spot beam with higher traffic demand and better channel condition will be allocated more resources to minimize the objective function. Due to the delay constraint in each spot beam, there was a minimal traffic demand in each spot beam.
TABLE 5: The total system capacity of different objective functions.

\begin{tabular}{lc}
\hline Order of objective function & $\sum C_{i}$ \\
\hline OBOP $n=2$ & $792.48124 \mathrm{Mbps}$ \\
OBOP $n=3$ & $792.48123 \mathrm{Mbps}$ \\
OBOPND $n=2$ & $792.48123 \mathrm{Mbps}$ \\
OBOPND $n=3$ & $792.44466 \mathrm{Mbps}$ \\
\hline
\end{tabular}

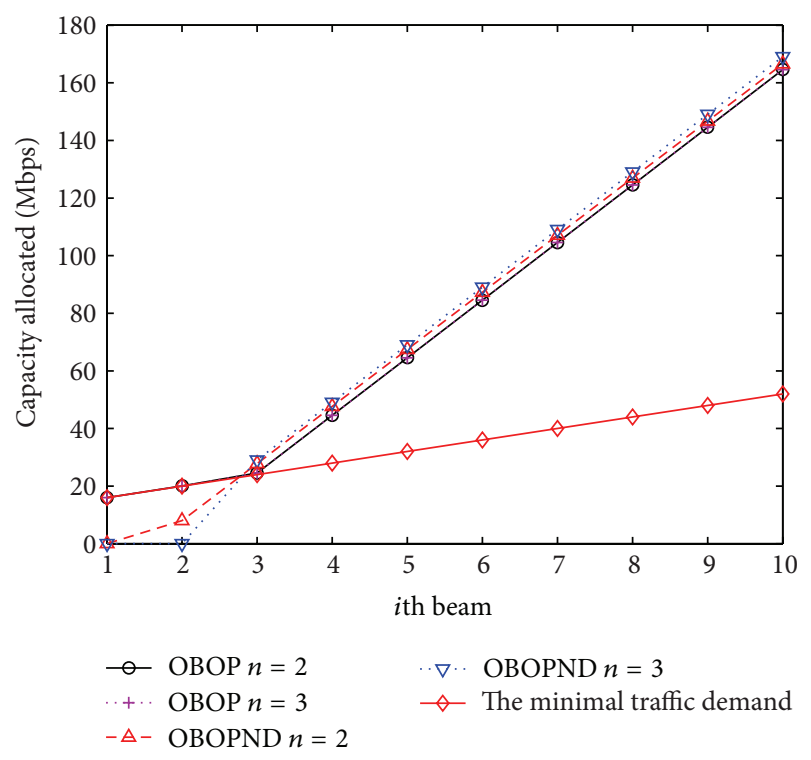

FIGURE 8: The capacity allocated to each spot beam of different objective functions.

When the channel condition of each spot beam was the same, the delay constraint only had an impact on the fairness among spot beams. However, when the channel conditions were not the same, both the total system capacity and fairness among spot beams were influenced by the delay constraint.

\section{Appendix}

We first prove that constraint (4) can be ignored by contradiction. Assuming that $C_{i}>T_{i}$, it is seen that the constraint (7) is satisfied obviously. As a result, the corresponding Lagrange multiplier $\rho_{i}$ is zero. According to (11), the value of $\lambda$ is negative, which contradicts that $\lambda$ is nonnegative. Therefore, the constraint (4) is satisfied when the value of $\lambda$ is nonnegative.

According to [14], for the optimization problem which has the following general form,

$$
\begin{array}{ll}
\min & f(\mathbf{X}), \\
\text { s.t. } & g_{i}(\mathbf{X}) \leq 0,
\end{array}
$$

when the functions $g_{i}(\mathbf{X})$ and $f(\mathbf{X})$ are convex, the optimization problem is convex. 
Taken together with the fact that the functions $\sum P_{i}$ and $\sum W_{i}$ in constraints (5) and (6) are linear, to prove the optimization problem with respect to $\left[W_{1}, W_{2}, \ldots, W_{n}\right.$, $\left.P_{1}, P_{2}, \ldots, P_{n}\right]$ is convex we only need to prove that the objective function $\sum\left(T_{i}-C_{i}\right)^{2}$ is convex and the function $C_{i}=W_{i} \log _{2}\left[1+\alpha_{i}^{2} P_{i} /\left(W_{i} N_{0}\right)\right]$ in constraint (7) is concave. Firstly, we prove that function $C_{i}$ with respect to $W_{i}$ and $P_{i}$ is concave.

The Hessian of the function $C_{i}=W_{i} \log _{2}\left[1+\alpha_{i}^{2} P_{i} /\right.$ $\left.\left(W_{i} N_{0}\right)\right]$ is given as follows:

$$
H_{C}=\left[\begin{array}{cc}
\frac{\partial^{2} C_{i}}{\partial P_{i}^{2}} & \frac{\partial^{2} C_{i}}{\partial P_{i} W_{i}} \\
\frac{\partial^{2} C_{i}}{\partial P_{i} W_{i}} & \frac{\partial^{2} C_{i}}{\partial W_{i}^{2}}
\end{array}\right]
$$

where $\partial^{2} C_{i} / \partial P_{i}^{2}=-\alpha_{i}^{2} W_{i} / \ln 2\left(N_{0} W_{i}+\alpha_{i}^{2} P_{i}\right)^{2} \leq 0$ and $\left|H_{C}\right|=0$.

It is seen that the determinant of the $H_{C}$ is zero; therefore, $H_{C}$ is negative semidefinite and the function $C_{i}$ is concave [14]. Then we prove that $\sum\left(T_{i}-C_{i}\right)^{2}$ is convex:

It is known that the sum of convex functions is also convex [14]. Therefore, to prove that $\sum\left(T_{i}-C_{i}\right)^{2}$ is convex, we just need to prove the following function is convex:

$$
f\left(P_{i}, W_{i}\right)=\left(T_{i}-C_{i}\right)^{n},
$$

where $C_{i}=W_{i} \log _{2}\left(1+\alpha_{i}^{2} P_{i} / W_{i} N_{0}\right)$.

The Hessian of $f\left(P_{i}, W_{i}\right)$ is given as follows:

$$
H_{f}=\left[\begin{array}{ll}
\frac{\partial^{2} f\left(P_{i}, W_{i}\right)}{\partial P_{i}^{2}} & \frac{\partial^{2} f\left(P_{i}, W_{i}\right)}{\partial P_{i} \partial W_{i}} \\
\frac{\partial^{2} f\left(P_{i}, W_{i}\right)}{\partial P_{i} \partial W_{i}} & \frac{\partial^{2} f\left(P_{i}, W_{i}\right)}{\partial W_{i}^{2}}
\end{array}\right] .
$$

To prove that $H_{f}$ is positive semidefinite, we obtain the following equation:

$$
\begin{aligned}
\frac{\partial^{2} f\left(P_{i}, W_{i}\right)}{\partial P_{i}^{2}}= & n(n-1)\left(T_{i}-C_{i}\right)^{n-2}\left(\frac{\partial C_{i}}{\partial P_{i}}\right)^{2} \\
& -n\left(T_{i}-C_{i}\right)^{n-1} \frac{\partial^{2} C_{i}}{\partial P_{i}^{2}} \\
= & n(n-1)\left(T_{i}-C_{i}\right)^{n-2}\left(\frac{\partial C_{i}}{\partial P_{i}}\right)^{2} \\
& +n\left(T_{i}-C_{i}\right)^{n-1} \frac{W_{i}}{\ln 2\left(N_{0} W_{i} / \alpha_{i}^{2}+P_{i}\right)^{2}}, \\
\frac{\partial^{2} f\left(P_{i}, W_{i}\right)}{\partial W_{i}^{2}}= & n(n-1)\left(T_{i}-C_{i}\right)^{n-2}\left(\frac{\partial C_{i}}{\partial P_{i}}\right)^{2} \\
& +n\left(T_{i}-C_{i}\right)^{n-1} \frac{\partial^{2} C_{i}}{\partial W_{i}^{2}},
\end{aligned}
$$

$$
\begin{aligned}
\frac{\partial^{2} f\left(P_{i}, W_{i}\right)}{\partial P_{i} \partial W_{i}}= & n(n-1)\left(T_{i}-C_{i}\right)^{n-2} \frac{\partial C_{i}}{\partial P_{i}} \frac{\partial C_{i}}{\partial W_{i}} \\
& -n\left(T_{i}-C_{i}\right)^{n-1} \frac{\partial^{2} C_{i}}{\partial P_{i} \partial W_{i}}, \\
\left|H_{f}\right|= & \frac{\partial^{2} f\left(P_{i}, W_{i}\right)}{\partial P_{i}^{2}} \frac{\partial^{2} f\left(P_{i}, W_{i}\right)}{\partial W_{i}^{2}} \\
& -\frac{\partial^{2} f\left(P_{i}, W_{i}\right)}{\partial P_{i} \partial W_{i}} \frac{\partial^{2} f\left(P_{i}, W_{i}\right)}{\partial P_{i} \partial W_{i}} \\
= & n^{2}(n-1)\left(T_{i}-C_{i}\right)^{2 n-3} \\
& \times\left[2 \frac{\partial C_{i}}{\partial P_{i}} \frac{\partial C_{i}}{\partial W_{i}} \frac{\partial^{2} C_{i}}{\partial P_{i} \partial W_{i}}-\left(\frac{\partial C_{i}}{\partial P_{i}}\right)^{2} \frac{\partial^{2} C_{i}}{\partial W_{i}^{2}}\right. \\
& \left.-\left(\frac{\partial C_{i}}{\partial W_{i}}\right)^{2} \frac{\partial^{2} C_{i}}{\partial P_{i}^{2}}\right]+n^{2}\left(T_{i}-C_{i}\right)^{2 n-2} \\
& \times\left[\frac{\partial^{2} C_{i}}{\partial W_{i}^{2}} \frac{\partial^{2} C_{i}}{\partial P_{i}^{2}}-\frac{\partial^{2} C_{i}}{\partial P_{i} \partial W_{i}} \frac{\partial^{2} C_{i}}{\partial P_{i} \partial W_{i}}\right] \\
= & n^{2}(n-1)\left(T_{i}-C_{i}\right)^{2 n-3} \\
& \times \frac{C_{i}^{2}}{W_{i} \ln 2\left(N_{0} W_{i} / \alpha_{i}^{2}+P_{i}\right)^{2}} .
\end{aligned}
$$

When $T_{i} \geq C_{i}$, it is obvious that (A.5) and (A.8) are nonnegative. Therefore, $H_{f}$ is positive semidefinite and $\sum\left(T_{i}-C_{i}\right)^{2}$ is convex. Taken together with the conclusion that $C_{i}$ is concave, we prove that the optimization problem is convex.

\section{Conflict of Interests}

The authors declare that they do not have any commercial or associative interest that represents a conflict of interests in connection with the work submitted.

\section{Acknowledgment}

The authors would like to thank the National High-Tech Research \& Development Program of China (863 Program) under Grant 2012AA01A508 for the project support.

\section{References}

[1] J. P. Choi and V. W. S. Chan, "Optimum power and beam allocation based on traffic demands and channel conditions over satellite downlinks," IEEE Transaction on Wireless Communication, vol. 4, no. 6, pp. 2983-2993, 2005.

[2] Y. Hong, A. Srinivasan, B. Cheng, L. Hartman, and P. Andreadis, "Optimal power allocation for multiple beam satellite systems," in Proceedings of the IEEE Radio and Wireless Symposium, pp. 823-826, January 2008. 
[3] F. Qi, L. Guangxia, F. Shaodong, and G. Qian, "Optimum power allocation based on traffic demand for multi-beam satellite communication systems," in Proceedings of the 13th International Conference on Communication Technology (ICCT '11), pp. 873-876, September 2011.

[4] U. Park, H. Wook Kim, D. Sub Oh, and B. J. Ku, "Optimum selective beam allocation scheme for satellite network with multi-spot beams," in Proceedings of the 4th International Conference on Advances in Satellite and Space Communications (SPACOMM '12), pp. 78-81, April2012.

[5] U. Park, H. W. Kim, D. Sub Oh, and B. J. Ku, "A dynamic bandwidth allocation scheme for a multi-spot-beam satellite system," ETRI Journal, vol. 34, no. 4, pp. 613-616, 2012.

[6] K. Kumaran and H. Viswanathan, "Joint power and bandwidth allocation in Downlink transmission," IEEE Transaction on Wireless Communications, vol. 4, no. 3, pp. 1008-1016, 2005.

[7] X. Gong, S. A. Vorobyov, and C. Tellambura, "Joint bandwidth and power allocation with admission control in wireless multiuser networks with and without relaying," IEEE Transactions on Signal Processing, vol. 59, no. 4, pp. 1801-1813, 2011.

[8] J. Miao, Z. Hu, K. Yang, C. Wang, and H. Tian, "Joint power and bandwidth allocation algorithm with Qos support in heterogeneous wireless networks," IEEE Communications Letters, vol. 16, no. 4, pp. 479-481, 2012.

[9] T. M. Cover and J. A. Thomas, Elements of Information Theory, John Wiley \& Sons, New York, NY, USA, 1991.

[10] G. Ding, Q. Wu, and J. Wang, "Sensing confidence levelbased joint spectrum and power allocation in cognitive radio networks," Wireless Personal Communications, vol. 72, no. 1, pp. 283-298, 2013.

[11] W. Yu and L. Raymond, "Dual methods for nonconvex spectrum optimization of multicarrier systems," IEEE Transaction on Communications, vol. 54, no. 7, pp. 1310-1322, 2006.

[12] R. Wang, K. N. Vincent Lau, L. Lv, and B. Chen, "Joint crosslayer scheduling and spectrum sensing for OFDMA cognitive radio systems," IEEE Transaction on Wireless Communications, vol. 8, no. 5, pp. 2410-2416, 2009.

[13] A. G. Marques, X. Wang, and G. B. Giannakis, "Dynamic resource management for cognitive radios using limited-rate feedback," IEEE Transactions on Signal Processing, vol. 57, no. 9, pp. 3651-3666, 2009.

[14] S. Boyd and L. Vandenberghe, Convex Optimization, Cambridge University Press, Cambridge, UK, 2004. 


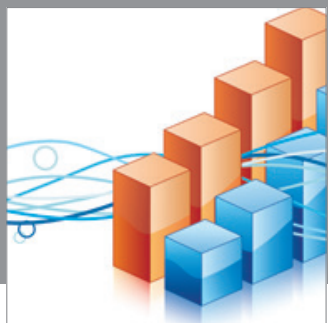

Advances in

Operations Research

mansans

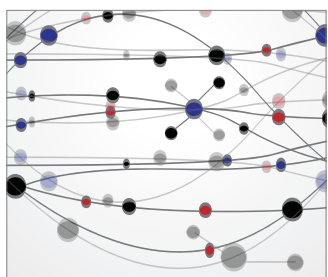

The Scientific World Journal
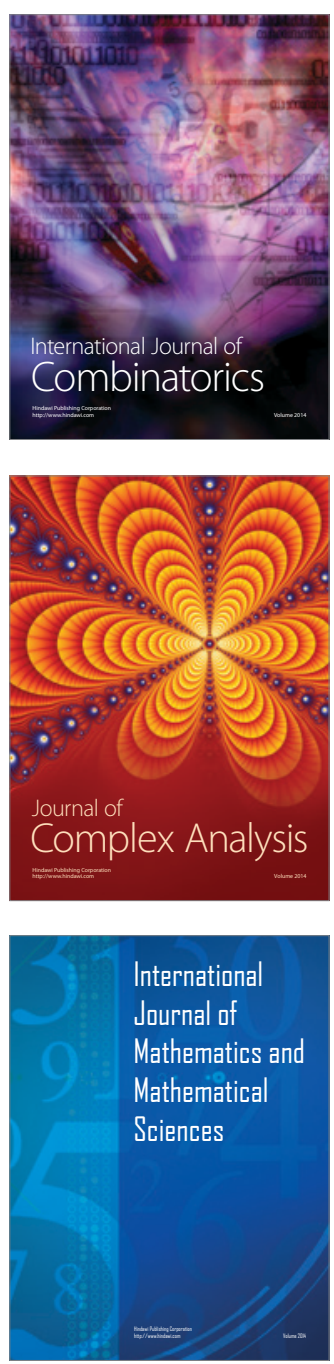
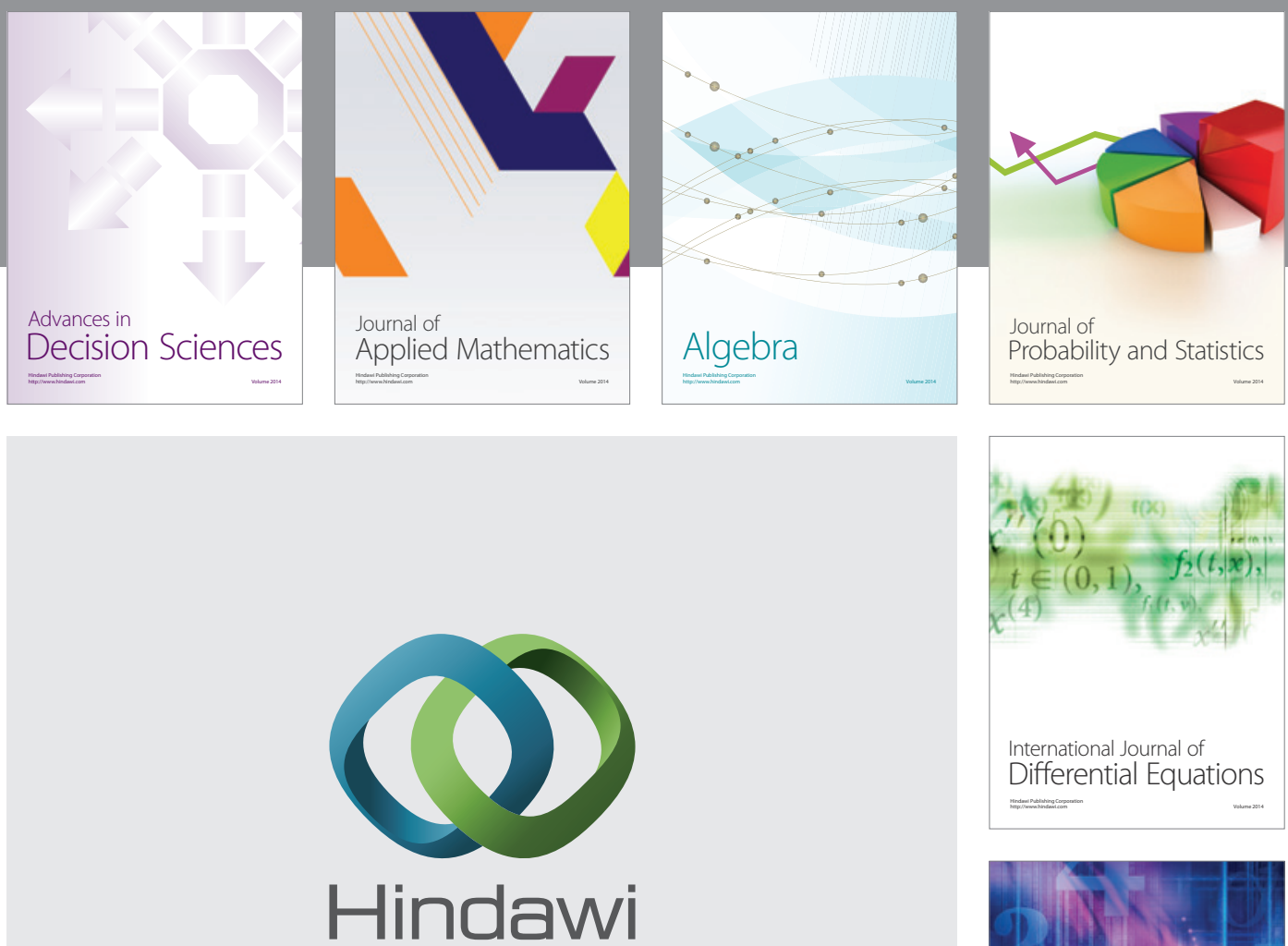

Submit your manuscripts at http://www.hindawi.com
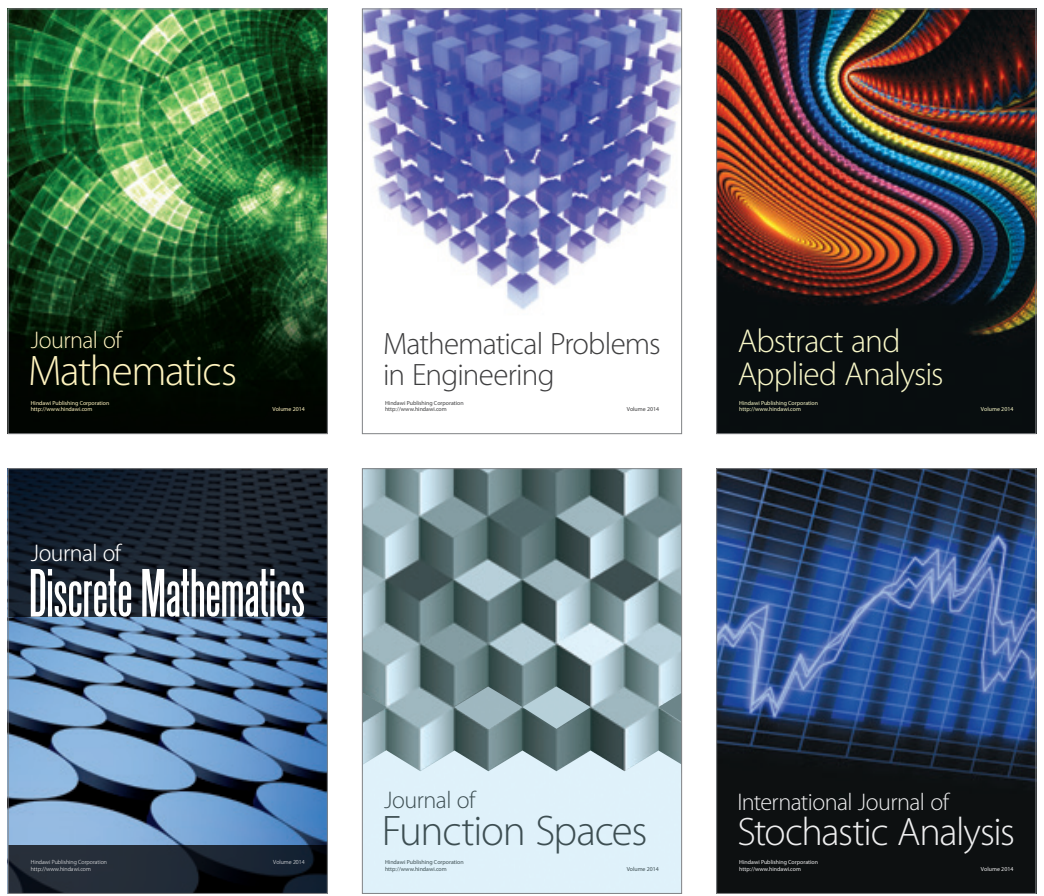

Journal of

Function Spaces

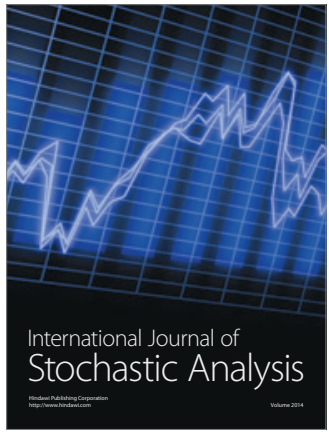

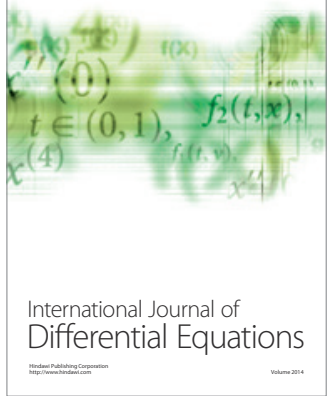
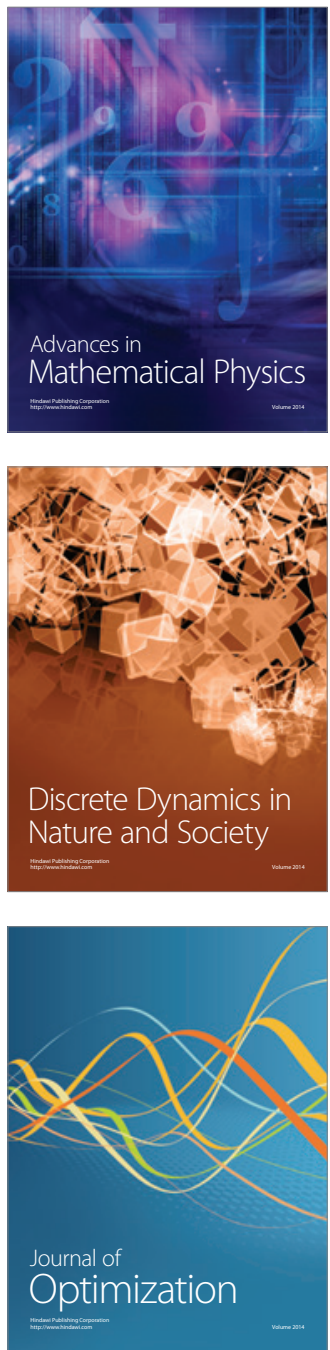